\title{
Fast Ion-Atom and Ion-Molecule Collisions
}




\title{
Interdisciplinary Research on Particle Collisions and Quantitative Spectroscopy
}

ISSN: 2315-4233

Series Editor: Dževad Belkić

\author{
Vol. 1: Fast Ion-Atom and Ion-Molecule Collisions \\ edited by Dž. Belkić
}

\author{
Forthcoming \\ Vol. 2: Fast Collisions of Light Ions with Matter: Charge Exchange and \\ Ionization \\ by Dž. Belkić
}




\section{Fast lon-Atom and \\ Ion-Molecule Collisions}

\section{Editor \\ Dževad Belkić}

Karolinska Institute, Stockholm, Sweden 
Published by

World Scientific Publishing Co. Pte. Ltd.

5 Toh Tuck Link, Singapore 596224

USA office: 27 Warren Street, Suite 401-402, Hackensack, NJ 07601

UK office: 57 Shelton Street, Covent Garden, London WC2H 9HE

\section{British Library Cataloguing-in-Publication Data}

A catalogue record for this book is available from the British Library.

Interdisciplinary Research on Particle Collisions and Quantitative Spectroscopy - Vol. 1 FAST ION-ATOM AND ION-MOLECULE COLLISIONS

Copyright $\odot 2013$ by World Scientific Publishing Co. Pte. Ltd.

All rights reserved. This book, or parts thereof, may not be reproduced in any form or by any means, electronic or mechanical, including photocopying, recording or any information storage and retrieval system now known or to be invented, without written permission from the Publisher.

For photocopying of material in this volume, please pay a copying fee through the Copyright Clearance Center, Inc., 222 Rosewood Drive, Danvers, MA 01923, USA. In this case permission to photocopy is not required from the publisher.

ISBN 978-981-4407-12-0

Printed in Singapore. 


\section{Editorial}

\section{Vision of interdisciplinarity within this book series}

It is instructive to inquire about the real meaning of interdisciplinary research within a given context, such as a book series. In general, besides obvious obstacles such as evident differences in the attitudes to problem solving and terminology, there is also the more subtle issue of mentality and barriers when experts from different research fields envisage to join forces. It would not be a rare event if a theoretical physicist would wish, from the start, to venture to tell, e.g. a potentially collaborating biologist what is actually important to study in biology. A biologist might attempt to view physicists or mathematicians alike as those who merely provide the needed service to biology. Both approaches are wrong, as they evidently stem from the lack of genuine appreciation of the special aspects and potential of the other branches of research. Some research physicists might even be tempted to think of going to another hardly necessary extreme of studying, e.g. all of biomedicine or vice versa, as if this were a pre-requisite for a successful interdisciplinary cooperation.

A more appropriate approach to a good cooperation of experts from different fields would seem to be striking a balance in acquiring the necessary minimum information about each other's expertise within the concrete problems under study, then to ask the right questions and finally to know what is at stake. It is within this general and realistic realm of viewing interdisciplinarity that this series is planned to encourage cooperative approaches to complex problems to be reviewed through different volumes of varying levels of complexity.

Specifically, this series is aimed at giving expert coverage to cuttingedge advances in studies of collisions and spectroscopy with a focus on prospects for interdisciplinary applications in the sense to which we alluded above. These two main strategies for investigating the structure of matter on 
various fundamental levels are deeply and intrinsically intertwined through a panoply of similar or common concepts as well as via mathematical and computational methods, of both a deterministic and stochastic nature.

The notion of spectroscopy naturally emerges from resonant scattering phenomena when unstable, decaying states are involved. Regarding collisions, the series will cover both light and heavy particle laser-free and laser-assisted collisions from low to high energies. This is rooted in the key interdisciplinary significance of such collisions in wide applications, including plasma physics, astrophysics, thermonuclear fusion research, radiotherapy, etc. The series will encourage contributions from basic as well as applied sciences, the joint home of both collisions and spectroscopy.

Over a long period of time, collisions and spectroscopy from basic research in physics and chemistry have made gigantic strides across interdisciplinary fields, including the life sciences. For example, nuclear magnetic resonance spectroscopy from fundamental research in physics and chemistry is viewed by experts to possess the potential of revolutionizing particularly early cancer diagnostics, molecular image-guided surgery and radiotherapy as well as follow-up.

Likewise, energetic collisions involving multiply-charged heavy particles are of paramount importance, as they are nowadays going through a veritable renaissance in several leading strategies, such as hadron therapy, fusion research, etc. High-energy light ion beams from protons to carbon nuclei, as a powerful part of hadron therapy of deep-seated tumors, are increasingly in demand worldwide, and this highly motivates construction of hospital-based accelerators. Positron-emitting secondary ions generated from collisions of primary projectiles with the treated tissue provide the possibility for special tomographic verifications of dose depositions at the targeted sites. Such information is of utmost importance, since any inaccuracy in dose delivery to the tumor relative to the prescribed dose planning system can be used to update the input data to the algorithmic codes for energy losses in order to provide the corrected doses in the subsequent fractionated treatments of the patient. This adaptive dose delivery and the proper inclusion of the so-called relative biological effectiveness are among the major constituents of biologically-optimized and, indeed, personalized radiotherapy which is adapted to the specific needs of each patient.

Collision physics involving ions is topical again in the quest for new energy sources, as greatly boosted by the International Thermonuclear Reactor (ITER) which is currently being built in France and shall be put 
into operation in 2020. This type of energy stems from high-temperature fusion of light nuclei and the main significance of this achievement is not just in a commercially profitable energy gain, but, most importantly, in having an incomparably safer energy production than the one from nuclear fission, due to minimum radioactive waste and no runaway nuclear reactions. This was made possible by tremendous intra- and inter-disciplinary cooperative efforts involving ion collision physics, plasma physics, engineering and technology. For example, the most critical for steady energy production is plasma stability. Plasma, being comprised of hot charged ions, can readily be neutralized and, as such, lost for generation of the current. Neutralization can occur through collisions of ions with the reactor walls via electron capture from the material contained in the tokamak. Knowledge from atomic collision physics about the largest cross sections for electron capture for certain elements enabled elimination of such materials from the walls of the tokamak and thereby contributed substantially to plasma stabilization.

This series explores both direct and inverse problems in scattering and spectroscopy. The primary emphasis is on methodologies of proven validity simultaneously based on fundamental theoretical concepts and principles as well as on indispensable and thorough comparisons with experiments. There is a growing concern among e.g. medical physicists that critical input from theories of basic sciences is needed for going beyond phenomenological and empirical, data-driven modeling with no predictive power. Such models are still overwhelmingly in use in, e.g. radiobiology of cell survival as well as in cross section data bases for Monte Carlo simulations of energy losses of ions during their passage through tissue. Critical and expert state-ofthe-art reviews of the existing progress and stringent assessments of the available methods in comparative analyses of theories versus experiments will be strongly encouraged.

Although physics was the main branch for studying particle collisions as well as to the major three spectroscopies (sub-nuclear, nuclear and atomic), mathematics was and still is pivotal to furthering these scientific strategies. This coupling resulted in the creation of a special field in mathematics per se, called "Scattering Theory". The themes of the series will nurture the ever needed inspiration from mathematics, both computational and fundamental, ranging from integral transforms through differential equations to inverse problems. The most interesting/important areas in theoretical and experimental studies across inter-disciplinary fields are within inverse problems of finding the causes from the observed effects. Only the simplest experiments in, e.g. physics deal with direct measurements of the sought 
observables. By contrast, in more fundamental physics experiments such observables are directly inaccessible, so that resorting to the concept of inverse problems is unavoidable by making inferences about the causes for the recorded effects. Likewise, and practically by definition, virtually the whole of medicine is about solving inverse problems. This is of paramount significance in practice and the series will embrace inverse scattering and spectroscopy problems that are plagued by notorious mathematical illconditioning and ill-posedness due to the lack of the models' continued dependence upon the analyzed data. Such inherent difficulties are further exacerbated by the presence of unavoidable noise.

Ubiquitous noise in experimental data and computationally generated spuriousness are invariably considered as a nuisance and detrimental to analysis and synthesis. This series will pay special attention to the modern advances in inverse collisional and spectroscopic problems where, surprisingly, noise can advantageously be exploited to reliably identify the sought physical and biochemical information and, thus, separate noise from true signal (stochastic resonances, Froissart doublets, etc.).

The series' interest in basic structure and overall interactions is aimed to offer the possibility for perceiving kindred relationships among apparently diverse systems in vastly different fields. This naturally includes system theory and optimization. System theory perceives the system's interactive dynamics as a whole, rather than merely its isolated constituent parts. Optimization comes into play to synergize the favorable and minimize the adverse influences of the surrounding environment of the system. This approach has already brought fruits to biology and medicine. For instance, optimization is a key to conformal radiotherapy which selectively targets the diseased tissue, while simultaneously sparing the healthy parts of the organ. In the like spirit of cross-fertilization, the self-contained field of signal processing is itself currently benefitting from the recently imported advances in quantum-mechanical spectral analysis. Here, regarding, e.g. medical diagnostics, mathematical optimization is also the most important, since experimentally encoded data are frequently uninterpretable in the direct domain in which measurements are performed.

There is not a single book or book series or journal which unifies spectroscopy and collisions into a common framework, as envisaged here. This series is designed to bridge this gap as a publication of the outlined unique profile. Such a scope is indispensable, given the inevitable scatter of information across interdisciplinary fields. The need to make bridges across 
various sources of spectroscopy and collisions is urgent, given the demonstrated potential of these universal methodologies for studying the structure of matter on vastly different levels/complexities. One of the rationale for establishing this type of interdisciplinary book series is to avoid duplication of methods and results. The lack of cross-disciplinary fertilization often leads to "discovering" some methodologies or techniques in one branch only to subsequently realize that it was merely a reinvention of the already known results from another branch of research and development.

The logo of the series is a symbol consisting of three intertwined rings that are known as Borromean rings in Mathematics and Effimov states in Physics. The latter symbolize a bound system of, e.g. three atoms where none of the three two-body sub-systems is bound. As such, the compound tripartite system breaks apart as soon as any of the sub-systems is removed. In the logo, the three rings represent symbolically Mathematics, Physics and Medicine, as the main backbone of the interdisciplinarity of this series. What is meant by this symbolism is that both Physics and Medicine are substantially enriched by their linkage to Mathematics, without which progress of either science would be most severely hampered, to say the least. Modern hospitals could not function without X-rays, computerized tomography, ultrasound, positron emission tomography, magnetic resonance imaging and magnetic resonance spectroscopy, all of which were brought to Medicine by Physics and Mathematics. The logo of the series places emphasis on the synergism achievable when these three disciplines are tightly bound together. This triple link is beneficial to Mathematics, as well. For example, the most difficult problems and questions raised particularly within Physics are already known as being capable of spurring fundamental progress in Mathematics. Critical advances on deeper levels in biomedical sciences also rely heavily upon mathematics due to the complexity of the problems and systems in the life sciences. It is likely that in the future, biomedical problems might also initiate research in yet unexplored pure mathematical avenues.

Overall, this series will focus on the multifaceted interdisciplinary character of collisions and spectroscopy. This is motivated by the existing wide consensus that the most profound progress in modern sciences of strategic importance for the society at large is critically guided by interand multi-disciplinary approaches to complex research problems.

By its versatile nature, the intended audience for this series is vast, including researchers and graduate students in physics, chemistry, biology, physicians of various levels of training, and the associated 
mathematical/computational sub-branches alongside the separate field of signal processing. The series will be published twice per year through independent books by one or more invited authors or edited volumes with chapters by different expert contributors.

Editor-in-Chief: Dževad Belkić Professor of Mathematical Radiation Physics Nobel Medical Institute, Karolinska Institute Stockholm, Sweden 


\section{Preface to Volume 1}

Volume 1 of this series is a collection of reviews on fast ion-atom and ion-molecule collisions. Here "fast" refers to the initial velocity or energy of the given projectile. Energetic ion beams are those with projectiles of speed considerably larger that the Bohr orbiting velocity of the target electron which is undergoing a transition from an initial to a final state. A typical reference energy of an ion beam is $25 \mathrm{keV} / \mathrm{amu}$ for a projectile nucleus of velocity $v=1 \mathrm{au}$, which matches the speed of the electron in the ground state of atomic hydrogen target. Relative to this reference energy, impact energies such as $25-400 \mathrm{keV} / \mathrm{amu}$ could be viewed as being in the intermediate region, whereas those above, e.g. $400 \mathrm{keV} / \mathrm{amu}$ might be considered as high incident energies. Volume 1 covers intermediate and high impact energies 25-7000 keV/amu of light ions (protons, alpha particles, lithium and carbon nuclei) colliding with atomic (helium) and molecular (water) targets. The investigated collisions are those that cause one- and two-electron transitions, such as single and double electron capture, single and double ionization, transfer ionization as well as simultaneous electron transfer and excitation of the residual target ion. Angular distributions of scattering projectiles and ejected electrons as well as total cross sections are among the main observables in both experiments and theories from Volume 1. Direct collaboration of experimentalists and theoreticians is always welcome and the results of such joint efforts occupy the first half of Volume 1 through chapters $1-4$. The remaining chapters $5-8$ are by theoreticians who likewise place their major attention on validation of their models against experimental data.

The experimental parts of chapters 1 and 2 are based upon one of the most powerful measuring devices from atomic and molecular collision physics called "cold target recoil ion momentum spectroscopy". This apparatus is a veritable "reaction microscope" which is capable 
of peering most deeply into the interactive dynamics of atomic and molecular collisions by way of kinematically complete experiments. Here, all the momenta of every actively participating particle can, in principle, be available, some of them by direct measurements and the others by reliance upon the momentum conservation law. This was made possible by exploiting the recoil target ion kinematics, which is usually left unexplored by, e.g. translational spectroscopy, due to conventionally unmeasurable small momenta. The rescue was in introducing the concept of target cooling, which enabled precise measurements of small recoil momenta, and this paved the road to kinematically complete experiments. With these achievements, theoreticians were offered the possibility for highly stringent testing of their methods on, e.g. fully differential cross sections and other similar observables.

As to theories from Volume 1, the challenges are multifaceted, especially at intermediate impact energies where the otherwise powerful perturbation methods are of limited applicability. On the other hand, non-perturbative methods are expected to be more adequate for this particular energy region, as also reviewed in Volume 1. The other level of challenges for theoreticians encompasses collisions with participation of two and more active electrons. Such collisions are characterized by electron-electron correlation effects that are known to play an important role at low and high energies, but have been reported in the past to be of minor significance at intermediate energies, as also analyzed in Volume 1. Yet another challenging aspect of the theories presented in this book is the area of molecular targets, among which water was chosen for illustration because of its pivotal role in medical physics. In medicine, and more precisely in radiotherapy, water is considered as a tissue-like matter because of its abundance in the human body. For this reason, most benchmark computations on ion energy losses in tissue, as needed for dose planning in radiotherapy, are carried out with water as a prototype tissue-like target. Here, the challenges are heightened by a large number of open channels, on top of the difficulties in handling the accompanying particle transport phenomena, such as energy as well as range fluctuations called straggling. One of the endpoints in this endeavor is to evaluate precisely the dose to be delivered by energetic ions at a given position located deeply in the treated body where the tumor resides. This is a very complex problem indeed, which demands many-layered expertise from nuclear and atomic collision physics. Part of Volume 1 is an initial contribution, which needs to be further pursued. 
In light of the above-outlined theme, our aim in Volume 1 is to present some of the leading classical, semi-classical and quantum-mechanical methods from ion-atom and ion-molecule collisions that could be relatively readily extended to a wider class of biomolecular targets of direct relevance to cancer therapy by energetic light ions. This is motivated by the intention of encouraging a systematic participation of a larger number of atomic and molecular collision physicists to the important problem of comprehensive and accurate determination of energy losses of ions when they traverse biomolecular targets, including tissue. This motivation is supported by the advantageous circumstance that the existing atomic collision physics methods can be adopted to biomolecular targets without undue difficulty, by using molecular wave functions in terms of linear combinations of Slatertype orbitals. This has indeed already been shown with the boundarycorrected first Born approximation and a simplified continuum distorted wave method. The next step would be to incorporate these methods into several powerful Monte Carlo simulations of energy losses of ions transported through tissue-like materials alongside their secondary particles (electrons and lighter nuclei). Very recently the first results along these lines have been reported.

The need for ions in radiotherapy stems from the most favorable localization of the largest energy deposition, precisely at the tumor site with small energy losses away from the target. Such a dose conformity to the target is due to heavy masses of ions that scatter predominantly in the forward cone and lose maximal energy only near the end of their path in the vicinity of the so-called Bragg peak, as illustrated in Fig. 1. The heavy masses of nuclei preclude multiple scattering of the primary ion beam. This occurrence is responsible for only about $30 \%$ of ion efficiency in killing tumor cells. However, ionization of targets by fast ions yields electrons that might be of sufficient energy to produce further radiation damage. These so-called delta-electrons can accomplish the missing $70 \%$ of tumor cell irradication mainly through multiple scattering enabled by the small electron mass. Therefore, energy depositions by both heavy (nuclei) and light (electrons) particles need to be simultaneously transported in Monte Carlo simulations. This is yet to be implemented in the existing codes.

Ions, electrons and photon beams are traditionally viewed as ionizing radiation modalities. This term "ionizing radiation" for cell kill is actually misleading, as it necessarily implies that only ionization is of relevance to radiotherapy as far as electromagnetic interactions are concerned. This is hardly the case, however, since radiation damage can be made by 


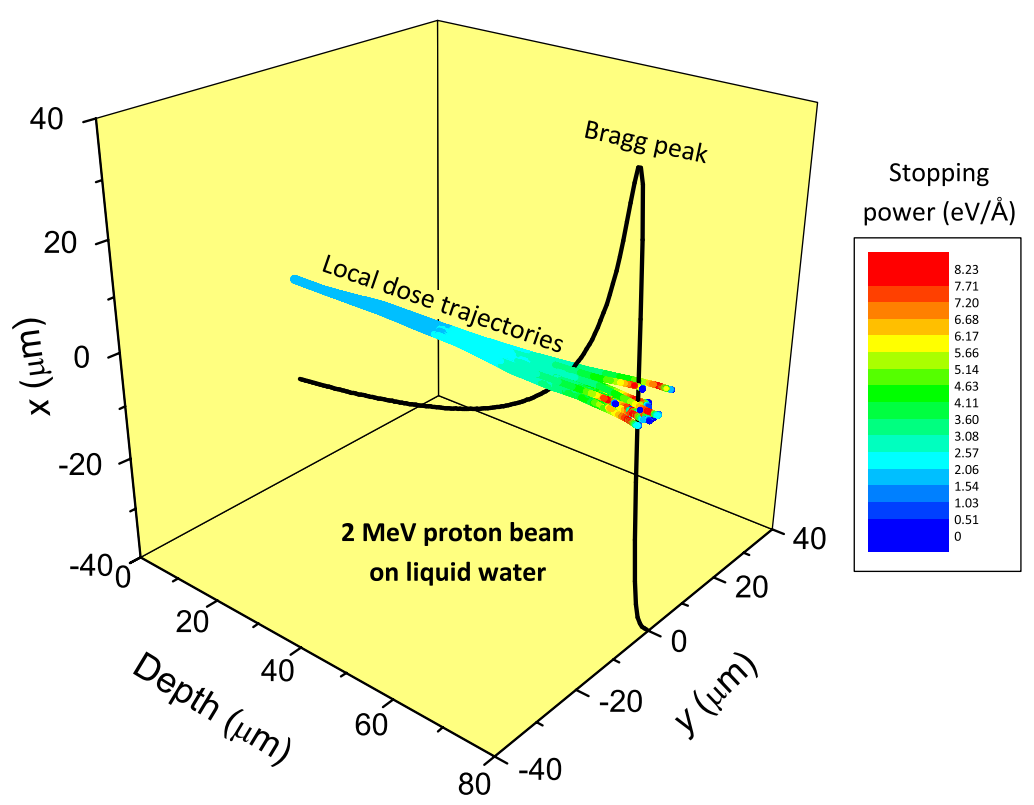

Fig. 1. Simulated trajectories and the associated local dose deposited by a $2 \mathrm{MeV}$ proton beam colliding with liquid water target. To avoid clutter, only a few trajectories are shown and the displayed depth-dose curve with the Bragg peak is qualitative. The figure is from chapter 8 with permission of the authors (Garcia-Molina et al.) who are hereby kindly thanked.

many other electronic processes such as excitation, electron capture, etc. Ionization and excitation are included in the Bethe-Bloch formula for stopping power, but not charge exchange. However, processes that govern charge states of projectiles, such as electron capture, are important when initially fast ions suddenly slow down near the Bragg peak. For example, $2 \mathrm{MeV}$ protons from the highlighted figure steadily keep the initial charge 1 for most of their track, but in the vicinity of the Bragg peak they easily become neutralized by electron capture from liquid water targets. The stopping power of the formed hydrogen atoms differs from that of protons. This implies that the Bethe-Bloch formula needs to be improved due to the missing charge exchange channel, which is important near the Bragg peak. The situation is actually more complicated because electron capture is promptly counteracted by electron loss, which is ionization of the formed hydrogen atoms in this case (or dressed ions in a general case). Only within the last few centimeters of the proton path, do literally electron capture and electron loss continuously and interchangeably take place thousands 
of times. Such a dynamic charge state of projectiles needs to be properly taken into account through, e.g. the appropriate rate equations. Instead, the common practice in Monte Carlo simulations is to use an empirical velocity-dependent screened nuclear charge of projectiles via the so-called Barkass effect. A number of other empirical formulae are employed in these stochastic algorithms for cross sections that are the most important input data for evaluation of ion energy losses. One of the goals of Volume 1 is to offer alternatives to these phenomenolgical approaches by presenting the perturbative and non-perturbative methods, both deterministic and stochastic (as well as their mixture) from atomic and molecular collision physics, with no adjustable parameters.

As per these remarks, there is plenty of room for improvement of the description of ion energy losses in matter, relative to the current situation encountered in, e.g. therapy by high-energy light ions. Part of the reason for relying heavily upon empirical cross section formulae in Monte Carlo codes for ions in hadron therapy is the lack of cross-talk between theory of atomic/molecular collisions and particle transport physics, when it comes to applied physics. One of the goals of Volume 1 is to bridge this gap and to initiate this type of cross-talk on a deeper and more fruitful level for the benefit of everyone involved, as per the spirit of this series.

When it comes particularly to hadron therapy, the interdisciplinarity of this series is inspired by the seminal work of physicist Robert Wilson who in the 1940s was the first to suggest in the medical journal Radiology, that protons, alpha particles or carbon nuclei and other light ions with their Bragg-peak-tailored dose distribution should provide optimal target conformity and tumor control. The significance of that proposal was in having been conveyed in such a convincing manner that physicians were quick to accept it. Moreover, physicians wasted no time in beginning patient treatments by light ions already in the 1950s. Such a unique example is admittedly hard to match, but this series will nevertheless strive whenever feasible to present the concepts and methodologies from one field in a way which should be relatively readily grasped and used by other research branches, including the life sciences.

- We will now go to the brief outlines of each of the chapters from Volume 1 with their most salient features.

Chapter 1 reviews recent studies on differential cross sections for double electron capture, as well as for simultaneous electron transfer and excitation of the residual target ion in proton-helium collisions at 
intermediate impact energies. Experimental data are compared to computations based upon perturbative and non-perturbative theories. The reported comparisons between experiment and theory show that the most interesting/important electron-electron correlation effects are difficult to identify in double capture and in transfer accompanied by target excitation in this energy region, because they are weak and masked by the projectileresidual target ion interaction.

Chapter 2 is on state-selective electron capture in collisions of alpha particles with helium targets at intermediate energies. State-selective capture mechanisms are identified from longitudinal momentum spectra. The recorded experimental data are compared with the classical trajectory Monte Carlo theory and good agreement is obtained at all the investigated energies.

Chapter 3 addresses the problem of double ionization as well as of single and double electron capture at intermediate energies. Two nonperturbative theories are employed, the Monte Carlo event generator and the coupled-channel basis generator methods. The independent particle formulation of the latter method is shown to work well even for double capture at the studied intermediate energies.

Chapter 4 presents several first-order four-body quantum-mechanical theories for fully differential cross sections in proton-helium collisions at intermediate energies involving single as well as double electron capture and also electron capture with target excitation. It was found that the electronelectron correlation effects in either the initial or final bound state have no appreciable influence on the computed angular distributions of scattered protons.

Chapter $\mathbf{5}$ deals with approximate three-body scattering states for the system of three charged particles that satisfy the correct asymptotic Coulomb boundary conditions for single electron capture as well as for direct and resonance ionization. It is shown that the interference of the distorted plane and spherical waves in scattering states can advantageously be used to analyze post-collisional interaction effects for spectra of autoionized electrons. Moreover, within the continuum intermediate state approximation, an illustration is given demonstrating that the classical Thomas double scattering could alternatively be described by interference between the distorted plane and spherical waves from the scattering Coulomb function.

Chapter 6 reviews total cross sections for several four-body collisional processes involving lithium nuclei and helium targets at intermediate as 
well as high impact energies. The possible reasons for the discrepancies among a number of theoretical predictions based upon classical and quantum mechanics are thoroughly discussed by reference to the available experimental data. Special attention has been paid to single and double electron capture, simultaneous transfer and ionization as well as to single and double ionization.

Chapter $\mathbf{7}$ is concerned with computations of cross sections for one- and two-electron processes in collisions of protons, alpha particles and carbon nuclei with water molecules. Studied are single electron capture, single ionization, double capture, transfer ionization and water fragmentation. Two methods are used, improved impact parameter classical trajectory Monte Carlo simulations and a semi-classical treatment with expansions in terms of molecular wave functions. A scaling law with respect to the projectile charge is derived at high energies and cross sections for molecular fragmentation after electron removal by single capture as well as single ionization are estimated.

Chapter 8 reports on the depth-dose profile and the spatial distribution of proton beams colliding with liquid water molecules. A combination of molecular dynamics and Monte Carlo simulations is used to investigate electronic stopping power, target electronic excitations, energy-loss straggling, elastic scattering as well the projectile charge-state due to electronic capture and electron loss processes. It is shown that electron capture becomes significant near the Bragg peak. Moreover, computations demonstrate that the position and the width of the Bragg peak is predominantly determined by the stopping power and energy straggling, respectively.

- Overall, as per chapters 1-8, this book, like any other book for that matter, is not a panacea for a straightforward knowledge transfer from one discipline to another. This is the case because, quite expectedly, there is no single theory, which would work universally by covering all the impact energies and all the collisions governed by electromagnetic interactions of light ions with atomic and molecular targets. Such theory is yet to come and this is the subject of active research in atomic and molecular collision physics. Nevertheless, for the purpose of, e.g. Medical Physics, Monte Carlo simulations of ion transport can still have a promising prospect from a judicious combination of the presented methods with their respective validity domains to cover most of the collisional phenomena of relevance to hadron therapy regarding electronic stopping power. This would be possible under the provision that a systematic extension to molecular 
targets, such as water molecules, will be soon accomplished e.g. along the lines discussed above or in some other alternative directions. Once this is achieved, pre-computations of cross sections from the best performing methods could be done to cover all the cases of interest in practice. This, in turn, would provide an invaluable modern recommended data base stored as a tabular interface from which fast and direct sampling could be made for Monte Carlo simulations. Such a procedure would make obsolete the ubiquitous reliance upon empirical formulae with fitting parameters adjusted to a limited set of the available experimental data. Some of the methods presented in Volume 1 are already near this sought operational stage, whereas others are to reach this status in the nearest future. It is hoped that Volume 1 of this series will spur a wider interest in the community of atomic and molecular collision physics for a more proactive participation to solving acute problems in Medical Physics with a particular focus on therapy by energetic light ions by using the available methodologies from Physics' first principles instead of phenomenological approaches.

Editor-in-Chief: Dževad Belkić Professor of Mathematical Radiation Physics Nobel Medical Institute, Karolinska Institute Stockholm, Sweden July 14, 2012 


\section{Acknowledgments}

We are grateful for the support from the Swedish Cancer Society Research Fund (Cancerfonden), the Radiumhemmet Research Fund, the Karolinska Institute Research Fund and the COST Action MP1002 "Nano-scale insight in ion beam cancer therapy (Nano-IBCT)". 
This page intentionally left blank 


\section{Contents}

Editorial $\quad \mathrm{v}$

Preface to Volume 1 xi

Acknowledgments $\quad$ xix

1. Electron Capture Processes in Ion-Atom Collisions at Intermediate Projectile Energies 1

M. Schulz, A. L. Harris, T. Kirchner and D. H. Madison

1. Introduction . . . . . . . . . . . . . . . . 2

2. Experimental Methods . . . . . . . . . . . . 5

3. Theory ........................ 7

4. Discussion ...................... 11

5. Conclusions . . . . . . . . . . . . . . 22

Acknowledgements . . . . . . . . . . . . . 24

References ....................... 24

2. COLTRIMS Experiments on State-Selective Electron

Capture in Alpha-He Collisions at Intermediate Energies $\quad 27$

M. Alessi, S. Otranto and P. Focke

1. Introduction . . . . . . . . . . . . . . 27

2. The COLTRIMS Concept . . . . . . . . . . . 30

3. Experiment . . . . . . . . . . . . . . . 33

4. Electron Capture in ${ }^{3} \mathrm{He}^{2+}+\mathrm{He} \ldots \ldots \ldots . \ldots . \ldots 37$

5 . Summary . . . . . . . . . . . . . . . . 51

References ........................... 51 
3. Recent Advances in the Theory and Modelling of Multiple

Processes in Heavy-Particle Collisions

T. Kirchner, M. Zapukhlyak, M. F. Ciappina and M. Schulz

1. Introduction . . . . . . . . . . . . . 56

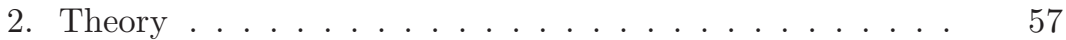

3. Results . . . . . . . . . . . . . . . 67

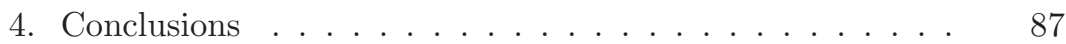

Acknowledgements . . . . . . . . . . . 88

References .................... 88

4. A 4-Body Model for Charge Transfer Collisions 93

A. L. Harris, J. L. Peacher and D. H. Madison

1. Introduction . . . . . . . . . . . . . . . . 93

2. General Theoretical Approach . . . . . . . . . . . . . 94

3. Four-Body Transfer with Target Excitation (4BTTE) Model ....................... 99

4. Four-Body Double Capture (4BDC) Model . . . . . . . . . 116

5. Conclusion . . . . . . . . . . . . . . . . 120

Acknowledgements . . . . . . . . . . . . 120

References .................... 121

5. Distorted Wave Methodologies for Energetic Ion-Atom Collisions

S. D. Kunikeev

1. Introduction . . . . . . . . . . . . . . . 123

2. Two-Body Coulomb Scattering . . . . . . . . . . . . . . . . . 125

3. Three-Body Coulomb Scattering . . . . . . . . . . . . . 148

4. Coulomb Scattering Effects in Ionization Electron Spectra . . . . . . . . . . . . . . . 160

5. Coulomb Scattering Effects in Autoionization Electron Spectra . . . . . . . . . . . . . . . 164

6. Effects of the Continuum Distortion in Charge Transfer . . 173

7. Discussion and Conclusions . . . . . . . . . . . . . . 181 Acknowledgements . . . . . . . . . . . . . . . 184

References . . . . . . . . . . . . . . . . . . 184 
6. Critical Assessment of Theoretical Methods for $\mathrm{Li}^{3+}$

Collisions with $\mathrm{He}$ at Intermediate and High

Impact Energies

189

Dž. Belkić, I. Mančev, and N. Milojević

1. Introduction . . . . . . . . . . . . . . . . . 189

2. Double Electron Capture . . . . . . . . . . . . . . . . . . 190

3. Single Electron Capture . . . . . . . . . . . . . . . . . 201

4. Transfer Ionization . . . . . . . . . . . . . . . . 204

5. Double Ionization . . . . . . . . . . . . . . . . . . . . . 215

6. Single Ionization . . . . . . . . . . . . . . . . . . . 222

7. Conclusions ...................... . . 224

Acknowledgements ................. . . 225

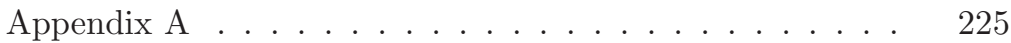

References ................... . . 226

7. Study of Inelastic Processes in Ion- $\mathrm{H}_{2} \mathrm{O}$ Collisions Using Classical Trajectory Monte Carlo and Semiclassical Methods

L. F. Errea, Clara Illescas, P. M. M. Gabás, L. Méndez,

I. Rabadán, A. Riera and B. Pons

1. Introduction . . . . . . . . . . . . . . . . . . . 231

2. Impact Parameter-CTMC Approach . . . . . . . . . . . . . 235

3. Semiclassical Calculations . . . . . . . . . . . . . . . 246

4. $\mathrm{H}^{+}+\mathrm{H}_{2} \mathrm{O}$ Collisions . . . . . . . . . . . . . . . . . 250

5. $\mathrm{He}^{2+}+\mathrm{H}_{2} \mathrm{O}$ Collisions . . . . . . . . . . . . . . 255

6. $\mathrm{C}^{6+}+\mathrm{H}_{2} \mathrm{O}$ collisions .................. . . 257

7. Scaling Laws and Fragmentation . . . . . . . . . . . . . . . 258

8. Conclusions ...................... 264

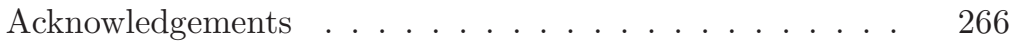

References .................. 266

8. Proton Beam Irradiation of Liquid Water: A Combined

Molecular Dynamics and Monte Carlo Simulation Study of the Bragg Peak Profile

R. Garcia-Molina, I. Abril, P. de Vera, I. Kyriakou and D. Emfietzoglou 
1. Introduction . . . . . . . . . . . . . . . . 272

2. Inelastic Energy-Loss Magnitudes of Swift Projectiles .................... 274

3. Simulation of Swift Particles Moving Through Condensed Matter . . . . . . . . . . . . . . . . 285

4. Results and Discussion . . . . . . . . . . . . . 292

5. Conclusions . . . . . . . . . . . . . . . . 299 Acknowledgements . . . . . . . . . . . 300 References .................. 300 $\begin{array}{ll}\text { Index } & 305\end{array}$ 